\section{Regards sur l'économie allemande}

Bulletin économique du CIRAC

79 | 2006

Varia

\title{
Modernisation graduelle du secteur bancaire allemand
}

Markus Gabel

\section{OpenEdition}

Édition électronique

URL : http://journals.openedition.org/rea/725

DOI : $10.4000 /$ rea. 725

ISBN : 978-2-8218-0854-6

ISSN : 1965-0787

\section{Éditeur}

CIRAC

\section{Édition imprimée}

Date de publication : 1 décembre 2006

Pagination : 21-28

ISSN : 1156-8992

\section{Référence électronique}

Markus Gabel, « Modernisation graduelle du secteur bancaire allemand », Regards sur l'économie allemande [En ligne], 79 | décembre 2006, document 3, mis en ligne le 01 décembre 2008, consulté le 21 avril 2019. URL : http://journals.openedition.org/rea/725 ; DOI : 10.4000/rea.725 


\section{Modernisation graduelle du secteur bancaire allemand}

\author{
Markus Gabel
}

Le secteur bancaire allemand est sorti de la forte crise du début du millénaire grâce à des restructurations et à des compressions d'effectifs sévères, ainsi qu'à l'embellie générale de l'économie. Mais le secteur a-t-il vraiment changé ? Certes, les banques allemandes ont évolué : structures plus modernes, reconfiguration des liens avec l'industrie, progrès de consolidation interne. Mais les grandes caractéristiques du secteur sont toujours là : dispersion en trois piliers (public, privé, coopératif) avec prépondérance des banques publiques, rentabilité médiocre et prédominance des banques dans le financement des entreprises. Ainsi, le secteur renoue largement avec la situation qui prédominait déjà vers la fin des années 1990. Mais même si l'opportunité, offerte par la crise, n'a pas été entièrement utilisée, le système bancaire allemand n'est pas condamné au statu quo. D'un côté, la Commission européenne persiste à remettre en question le système des trois piliers, de l'autre, la modernisation des structures de gouvernance financière progresse. La poursuite d'une sorte de réforme graduelle semble ainsi assurée. La question est de savoir jusqu'où celle-ci peut aller sans susciter une rupture systémique avec des conséquences inconnues.

Le secteur bancaire allemand est sorti de la sévère crise de 2002/2003. 2005 a été une année d'assainissement : les efforts de restructuration (licenciements drastiques et externalisation d'activités) ont porté leurs fruits, et les banques tirent profit de l'embellie macroéconomique nationale et internationale ; les dépenses se sont contractées et les recettes ont augmenté. En 2005, les banques allemandes ont pu à nouveau constituer des réserves. La situation devrait se stabiliser en 2006, et les perspectives restent positives: l'accélération de la croissance allemande qui touche non seulement les entreprises, mais aussi les consommateurs, offre aux établissements des marges naturelles de croissance. L'ensemble de ces phénomènes permet au secteur de normaliser sa rentabilité : si on met en relation le bénéfice global avec la somme des actifs du secteur, on obtient pour 2005 une rentabilité d'environ 0,5\% (depuis 1980, cette rentabilité se situe, à l'exception de la crise de $2002 / 03$, entre $0,5 \%$ et $1 \%$ ).

Mais si l'ensemble du secteur va mieux, l'amélioration est assez inégalement répartie : elle concerne davantage les banques privées, qui ont réalisé près de la moitié des bénéfices du secteur en 2005. Celui-ci retrouve ainsi une de ses caractéristiques traditionnelles : la forte variabilité des bénéfices du secteur privé va de pair avec la plus ou moins grande stabilité du secteur public et du secteur coopératif/mutualiste. Cette divergence provient du fait que les banques privées, mais aussi les Landesbanken, sont plus actives dans la banque de marché et sont ainsi plus exposées aux aléas des marchés comme l'éclatement de la bulle Internet. Par ailleurs, la crise du début du millénaire avait été accélérée par l'effondrement du marché immobilier allemand, ainsi que - et surtout par la dépréciation des portefeuilles de participation dans l'industrie, obligeant les banques à inscrire d'importantes provisions pour risques dans leurs bilans.

Si globalement le secteur renoue avec la profitabilité de la fin des années 1990, celle-ci reste largement inférieure à celle enregistrée par ses concurrents étrangers. Ailleurs, non seulement la crise a été moins profonde, mais surtout, la
Les banques allemandes sont sorties de la crise

Les banques privées renouent avec les bénéfices

Mais leur profitabilité reste faible 
Forte dispersion du marché

Caisses d'épargnes (local)

Landesbanken

Coopératives de crédits (local)

Caisses centrales coopératives

Grandes banques privées

Banques privées régionales et étrangères

Banques hypothécaires ${ }^{1)}$

Banques spéciales ${ }^{2)}$

Total

restructuration et la consolidation du marché sont plus avancées. Si en 2005, l'ensemble du secteur allemand a réalisé un bénéfice après impôts de 23,5 milliards $€$ (après 4,8 milliards $€$ en 2004 ), ce dernier dépasse pourtant à peine celui de la seule Citibank (19 milliards $€$ nets). La conséquence est que la plus grande banque allemande, la Deutsche Bank, dont le bénéfice s'élève à 3,5 milliards $€$, est loin du peloton de tête européen (12,6 milliards $€$ pour HSBC, 7,5 pour ING ou 4,5 pour la Société Générale).

La faible rentabilité est étroitement liée à l'autre caractéristique de fond du système bancaire d'outre-Rhin. Celui-ci est depuis toujours basé sur trois piliers : public, privé et coopératif. Cette structure, où deux acteurs (publics et coopératifs) pratiquent une forme de socialisation des bénéfices (les premiers pour des fins d'intérêt public, les deuxièmes envers leurs membres), est partiellement garantie par la loi, car la législation bancaire n'autorise pas le rachat de banques publiques par des acteurs privés. Les possibilités de croissance externes sont ainsi limitées et contribuent à la faible part de marché des banques privées (environ $18 \%$ du marché des clients domestiques, contre plus de $50 \%$ en France ou en Grande Bretagne). Cette dispersion du marché ne favorise guère des structures efficaces (par exemple en termes de nombre de filiales par tête ou de gestion du back office). Ainsi, le secteur bancaire allemand a davantage de difficultés à exploiter l'important potentiel d'économies d'échelles de l'industrie bancaire et à réduire le niveau élevé de coûts fixes.

Structure du système bancaire allemand à la fin 2005

\begin{tabular}{rc|rc|rc|}
\multicolumn{2}{|c|}{ Nombre de banques ${ }^{3)}$} & \multicolumn{2}{|c|}{ Nombre de filiales } & \multicolumn{2}{|c|}{ Actifs bancaires $\left.{ }^{3)} 4\right)$} \\
463 & $22 \%(19 \%)$ & 14801 & $40 \%$ & 1014 & $15 \%(16 \%)$ \\
12 & $1 \%(1 \%)$ & 618 & $2 \%$ & 1365 & $20 \%(20 \%)$ \\
1293 & $62 \%(68 \%)$ & 13357 & $37 \%$ & 592 & $9 \%(9 \%)$ \\
2 & $0 \%(0 \%)$ & 12 & $0 \%$ & 224 & $3 \%(4 \%)$ \\
4 & $0 \%(0 \%)$ & 2454 & $7 \%$ & 1227 & $18 \%(15 \%)$ \\
247 & $12 \%(10 \%)$ & 2421 & $7 \%$ & 706 & $10 \%(11 \%)$ \\
50 & $2 \%(1 \%)$ & 2903 & $8 \%$ & 1079 & $16 \%(14 \%)$ \\
18 & $1 \%(1 \%)$ & 27 & $0 \%$ & 969 & $10 \%(9 \%)$ \\
2089 & - & 36593 & - & 6903 & -
\end{tabular}

Sources : Deutsche Bundesbank, Rapport mensuel, septembre 2006 ; Gabel (2000). (1) Publiques et privées. (2) Etablissements à attributions spéciales, dont la Kreditanstalt für Wiederaufbau. (3) Les chiffres entre parenthèses renvoient à la situation en 1999. (4) En milliards $€$.

Modernisation de l'environnement et restructurations internes

Consolidation au sein de chacun des trois piliers

Une concurrence réduite au sein du système bancaire
Ce double constat (assainissement, mais non rattrapage des concurrents étrangers) peut étonner face aux efforts engagés ces dernières années. D'abord, on observe une modernisation touchant l'ensemble de l'environnement financier. Le droit financier et les règles de la gouvernance financières ont été modernisés, et le financement par le marché devient une réelle alternative pour les entreprises. Ensuite, en interne, les banques allemandes ont restructuré leurs liens avec l'industrie et elles se sont engagées dans un processus de réduction des coûts en comprimant leurs effectifs (80 000 personnes depuis 2000, essentiellement par licenciement), et en rationalisant leur organisation (externalisation des activités du back-office).

Enfin, le secteur enregistre également des progrès dans la consolidation interne aux piliers. Par exemple, la Landesbank Bade-Wurtemberg a intégré son homologue de Rhénanie-Palatinat ; la WestLB a repris la Weberbank ; la Commerzbank, a racheté l'Eurohyp, ce qui la classe au second rang des banques privées ; la Deutsche Bank s'est offert la Norisbank et plus récemment la Berliner Bank. Mais sur la scène européenne, les banques allemandes demeurent trop petites, trop peu rentables et dotées de cours de bourse trop faibles pour participer à la vague de consolidation européenne. Elles deviennent de ce fait des cibles comme l'a montré le rachat de HypoVereinsbank par l'italien UniCredit.

Si on considère le plus long terme, les récents changements ne pèsent pas très lourd. Outre-Rhin, les établissements bancaires sont toujours des banques uni- 
verselles (leur offre est peu spécialisée), le principe de la Hausbank (relation étroite entre l'entreprise et 'sa' banque) est toujours dominant, et le crédit bancaire reste, malgré la montée des modes de financement alternatifs, le moyen de financement prédominant pour les entreprises, notamment les PME. En même temps, les banques continuent à entretenir, quoique à moindre niveau, des liens capitalistiques avec l'industrie et restent présentes dans les organes de surveillance. Par ailleurs, la répartition des parts de marchés entre les trois piliers demeure très stable et, avec elle, la dispersion du secteur. Le système économique rhénan reste ainsi organisé autour d'un système bancaire lui assurant un haut degré de consistance, basé sur le principe de la complémentarité entre structures bancaires et économiques. Cependant, cette inertie systémique a des corollaires : non seulement, elle contribue à un nombre élevé d'établissements, mais surtout elle a tendance à réduire la concurrence à l'intérieur du système, car les très nombreuses caisses d'épargne et banques mutualistes ne se font pas de la concurrence entre elles, leur action étant limitée à une commune ou une ville par le principe régional (Regionalprinzip).

\section{Problème de rentabilité versus problème de coût}

Le problème de rentabilité des banques allemandes, notamment des banques privées, ne date pas de la crise de 2002/03. Les banques privées, quant à elles, le mettent habituellement sur le compte de la segmentation en trois piliers et de la concurrence «démesurée » exercée par les banques publiques. Mais cette vision est très partielle, comme le révèle la structure des bénéfices des banques allemandes. Comme l'a montré la Commission européenne en 2003, la marge d'intérêts nets, c'est-à-dire la différence entre les intérêts reçus et les intérêts payés, est particulièrement élevée en Allemagne. Depuis, elle s'est même encore accentuée en faveur des banques allemandes. Concrètement, les clients payent en moyenne leurs crédits plus cher outre-Rhin qu'ailleurs en Europe ( 0,7 point de pourcentage pour des crédits logement, 0,5 pour les crédits à la consommation et 0,3 pour les crédits longs aux entreprises).
Une marge d'intérêts nets particulièrement élevée...

Recettes bancaires par catégorie en Europe en $2005^{1)}$

\begin{tabular}{|c|c|c|c|c|c|}
\hline & Comptes courants & Dépôts & Crédit de consommation & Crédit immobilier & Cartes de crédit \\
\hline Allemagne & 186 & 676 & 504 & 1319 & 50 \\
\hline France & 124 & 36 & 410 & 1077 & 45 \\
\hline Grande Bretagne & 145 & 93 & 430 & 896 & 137 \\
\hline Italie & 204 & 67 & 337 & 1083 & 41 \\
\hline Pays-Bas & 108 & 64 & 705 & 617 & nd \\
\hline Autriche & 152 & 52 & 517 & 921 & nd \\
\hline Espagne & 40 & 101 & 363 & 1787 & 50 \\
\hline Moyenne EU-15 & 133 & 69 & 421 & 1126 & 64 \\
\hline
\end{tabular}

Source : Commission européenne, 2006, cité par le Handelsblatt, le 21 juillet 2006. (1) Recettes brutes par client en euro.

La première publication de ces chiffres en 2003 a été très mal reçue en Allemagne. En effet, elle intervenait au moment où la rentabilité des banques allemandes était au plus bas. La Bundesbank s'est même sentie obligée de remettre en question la comparabilité des chiffres. Mais l'actualisation des chiffres par la Commission en 2006 a confirmé l'impression précédente. Les banques elles-mêmes ont du mal à admettre ces faits. Plutôt, elles constatent une érosion de leurs marges d'intérêt net. En effet, depuis 2003, elles sont tombées en moyenne de 1,8 point de pourcentage à 1,2 (davantage pour les crédits immobiliers que pour les crédits aux entreprises).

Cette baisse, qui pourrait signaler une accélération de la concurrence entre les banques, ne doit pas faire oublier que les marges demeurent toujours plus élevées qu'à l'étranger. Le véritable problème de rentabilité n'est donc pas du côté des marges d'intérêt, mais bien du côté des coûts. Si on met en relation

... quoi qu'en disent les banques allemandes

Le problème de rentabilité provient des coûts, ... 
... surtout pour les banques privées une nouvelle logique de groupe

II contrôle plus d'un tiers du marché

Banques coopératives : pas de stratégie de groupe possible recettes et coûts (gestion du back office, administration interne, coût d'exploitation des agences), on constate que pour chaque euro de recettes gagné, les instituts allemands supportent des coûts pouvant aller jusqu'à 80 cents, nettement plus que leurs homologues européens (par exemple : 41,8 cents pour la Royal Bank of Scotland, 61,2 cents pour BNP Paribas, mais 74,7 et 89,4 cents pour la Deutsche Bank et la Dresdner Bank).

Depuis longtemps, la banque de détail pose un problème particulier. En moyenne, $75 \%$ des recettes y sont absorbées par des coûts internes. C'est le plus mauvais résultat parmi les quatre plus grands pays européens. Du côté des banques privées, les coûts sont particulièrement élevés. Les autres secteurs bancaires sont plus efficaces, notamment les caisses d'épargne. Ces chiffres expliquent pourquoi la rentabilité moyenne des banques privées reste depuis 2000 loin derrière celle des autres deux secteurs. C'est seulement au niveau du cross selling, donc de l'efficacité du marketing, que les banques privées se situent au même niveau que les deux autres secteurs. En comparaison européenne, ce niveau est cependant très bas. Alors que les concurrents européens parviennent à vendre en moyenne 4 autres produits à un client qui souscrit un crédit immobilier, les banques allemandes n'arrivent qu'à 2,2.

\section{Les trois piliers axent tout sur la croissance interne}

Compte tenu de ces problèmes de rentabilité, chacun des trois piliers poursuit sa propre stratégie, mais tous favorisent la croissance interne. Dans ce contexte, les banques publiques restent en position de force malgré la perte de leurs garanties formelles en 2005 et les nouvelles démarches de la Commission européenne (au sujet du nom de «Sparkasse »). Avec un bilan chiffré à 3300 milliards €, le Sparkassen Finanzgruppe (composé notamment de 463 caisses locales, de 12 compagnies d'assurance, de 11 Landesbanken, de 11 sociétés d'épargne immobilière) est de fait la plus grande banque au monde. Un tel raisonnement en termes de groupe devient désormais réalité, car la fédération des caisses, la DSGV, a abandonné son traditionnel concept basé sur la décentralisation. Ainsi, au lieu de laisser, comme dans le passé, l'autonomie totale à chaque caisse locale, le groupe axe tout sur une offre homogène, commercialisée dans l'ensemble du pays sous les mêmes conditions. Le début a été fait cette année avec un crédit à la consommation et dans le financement immobilier. D'autres produits sont en préparation (financement des PME, polices d'assurance, gestion des portefeuilles de la clientèle privée aisée).

L'objectif est d'accroître une part de marché déjà confortable : $37 \%$ de l'argent des ménages est géré par le secteur public, contre seulement 18,1\% par les quatre grandes banques privées, talonnées de près par le secteur coopératif $(17,4 \%)$. Outre l'homogénéisation de l'offre, le groupe progresse également sur un autre chantier : mieux intégrer les Landesbanken (établissements régionaux) dans les activités du groupe, car une coopération plus étroite entre caisses locales et régionales est un levier pour compenser la perte des garanties et maintenir les conditions de refinancement à un niveau favorable. Compte tenu de la taille du groupe, cette double stratégie dispose d'un énorme potentiel. Et elle a une implication directe pour tout le secteur bancaire allemand : avec un groupe qui contrôle à lui seul plus d'un tiers du marché, le niveau de concurrence interne au système bancaire allemand n'est guère renforcé.

Au contraire des caisses d'épargnes, les banques coopératives et mutualistes rencontrent de réelles difficultés. Pourtant, ce secteur a pris des mesures drastiques : aujourd'hui, il ne reste plus que deux établissements centraux et 1293 coopératives de crédit locales (par rapport à respectivement quatre et 2035 en 1999). Parallèlement, la part du secteur coopératif dans le total de l'actif bancaire est tombée de $13,1 \%$ à $12 \%$. Le problème du secteur est d'ordre structurel. Depuis les fusions de 2000 et 2001, la DZ Bank à Francfort et la WGZ Bank 
à Düsseldorf forment la tête du groupe. Leur capital est détenu par les coopératives de crédit locales (la base) qui non seulement sont autonomes et insistent sur leur indépendance, mais déterminent également la stratégie du groupe. On est donc à l'opposé du top-down tel qu'il est pratiqué par le concurrent public, les caisses d'épargnes et Landesbanken.

L'autre problème du secteur coopératif est l'insuffisance des capitaux. Actuellement, chaque augmentation de capital dépend de la bienveillance de la base : les coopératives locales. La fusion entre DZ et WGZ Bank, ainsi qu'une introduction en bourse d'un des établissements centraux, pourraient remédier à ce problème. Mais pour l'instant, les petites coopératives sont attachées à leur autonomie. Une introduction en bourse n'a ainsi une chance de réussir que si la majorité des capitaux reste aux mains des coopératives locales. La différence avec le secteur coopératif français est frappante : non seulement Natexis, son établissement central, est depuis longtemps coté en bourse, mais le secteur coopératif et les caisses d'épargnes ont fusionné leurs banques d'investissement Natexis et Ixis pour former Natixis. Une telle opération fait rêver outre-Rhin. En effet, un holding qui réunirait les établissements centraux et laisserait la clientèle privée aux caisses d'épargnes et coopératives locales pourrait former un géant bancaire avec des actifs de plus de 1000 milliards $€$, dépassant ainsi la Deutsche Bank.

Du côté des grandes banques privées, la situation est très ambiguë. D'un côté, il y a bien sûr la Deutsche Bank, seul véritable champion international, mais de l'autre, les instituts privés peinent à atteindre une taille critique. Après le rachat de HBV, les possibilités de fusions sont extrêmement limitées car il ne reste plus que la Deutsche Bank, la Commerzbank et la Postbank. Faute de possibilités de croissance externe, et après de nombreuses erreurs stratégiques (coûteuses excursions dans la banque d'affaires, allocation de crédits sans véritable prise en compte des risques, faible degré de spécialisation dans l'offre de produits), les banques privées visent également la croissance interne. La clientèle des particuliers est au centre la nouvelle stratégie et avec elle, la traditionnelle agence bancaire : le nombre d'agences a augmenté pour la première fois depuis 1991. L'augmentation a été la plus forte du côté des grandes banques privées $(+9 \%)$. La raison de cette renaissance est la redécouverte de la filiale comme canal privilégié de distribution. Les acquisitions récentes de la Deutsche Bank témoignent de cette « nouvelle stratégie ».

Ce revirement tranche nettement avec le passé où les banques privées avaient axé leur expansion sur la banque d'investissement; la Deutsche Bank voulait même se défaire de son activité banque de détail. Mais le succès inattendu de plusieurs banques étrangères sur le marché allemand a contribué à changer la donne. Ce succès a été facilité par une erreur de stratégie de la part des banques privées lors du collapsus de la « nouvelle économie ». Suite à la fermeture de nombreuses filiales des banques privées, les opérateurs étrangers ont saisi la brèche avec des offres particulièrement agressives.

Les banques privées visent un pan d'activité en particulier : le crédit à la consommation, négligé jusque-là au profit des caisses d'épargne, du secteur mutualiste, de certains établissements spécialisés comme la Norisbank, mais aussi des établissements étrangers. L'actuel regain de la confiance des consommateurs, ainsi que l'augmentation de la TVA en 2007, font que cette activité devient réellement stratégique. Elle est l'activité la plus rentable après les crédits immobiliers. Actuellement, les banques privées (15\% des actifs bancaires) ne couvrent que $8 \%$ du marché. Les caisses d'épargne, les banques régionales et le secteur coopératif et mutualiste détiennent plus de $80 \%$ du marché (respectivement $34 \%$, $28 \%$ et $22 \%$ ).

Bien que les banques privées doivent ainsi combler leur retard pour faire face à la place acquise par des nouveaux entrants (établissements étrangers), les cartes peuvent être redistribuées dans la banque de détail. Actuellement, le
Insuffisance des capitaux due à la trop forte l'autonomie des coopératives locales

Banques privées : redécouverte de la clientèle des particuliers...

... sous la pression de la concurrence étrangère

Objectif stratégique : le crédit à la consommation

Banque de détail : avantage aux Sparkassen 
Un regain de concurrence en perspective
Les Landesbanken n'ont perdu leurs garanties qu'en apparence

Des signes d'ouverture à la concurrence..

Quelle justification pour le statut public des caisses d'épargne? premier établissement bancaire est la Postbank avec près de 15 millions de comptes de particuliers, devant la Deutsche Bank (8,7 millions), la Dresdner Bank (5,8 millions) ; ING-Diba, la Commerzbank et la Citibank suivent avec respectivement $5,4,5$ et 3,1 millions de clients privés. Cependant, si on raisonne en termes de groupe, le constat est très différent: ainsi, le groupe public (caisses d'épargnes et Landesbanken) se situe loin devant avec environ 50 millions de comptes de particuliers!

Finalement, l'évolution des parts de marchés dépendra des offres proposées par les opérateurs. Une guerre des prix pourrait être la conséquence. Si certains parlent déjà d'une « Aldisation » du secteur bancaire - au moins en ce qui concerne la banque de détail -, il faut en tout cas admettre que cette redécouverte de la clientèle des particuliers pourrait enfin accentuer la concurrence sur le marché, limitée jusque-là par le fait que les banques privées avaient abandonné cette partie de la clientèle aux secteurs public et coopératif, opérant en plus selon le principe régional.

\section{Le statut des banques publiques sur la sellette}

Cependant, le véritable éveil de la concurrence ne pourra venir que d'une révision de la place des banques publiques. Sous la pression de la Commission européenne, les garanties publiques pour les caisses d'épargne et Landesbanken ont disparu le 18 juillet 2005. Cette date aurait pu être le début d'une réorganisation du système bancaire. Cependant, le contraire se produit, car le secteur public se renforce pour contrebalancer la perte des garanties : les Landesbanken font de l'expansion horizontale, et la fédération DSGV mise sur la centralisation via une spécialisation à l'intérieur du groupe. Par ailleurs, les garanties n'ont pris fin que sur le papier ; implicitement, elles perdurent. Les agences de rating, en effet, prennent en compte le fait que les propriétaires des banques publiques n'ont pas changé : ce sont toujours des collectivités publiques avec une autorité fiscale, reliées entre elles via un système de péréquation financière dans le cadre du fédéralisme financier allemand. Par conséquent, les notes de rating des Landesbanken n'ont guère varié depuis 2005 et demeurent nettement au-dessus de celles des banques privées. En outre, la politique exerce une protection réelle du secteur, comme l'a montré l'intervention en dernière minute du gouvernement du Mecklembourg afin d'empêcher la vente de la caisse d'épargne de Stralsund.

Le front de protection commence néanmoins à s'ouvrir. Suite au désastre financier de la Berliner Bankgesellschaft (BBG), la Commission européenne n'avait autorisé le généreux sauvetage public (soutenu financièrement par la Commission) qu'à la condition que la Berliner Sparkasse, appartenant à la BBG, soit vendue au plus tard en 2007, et ceci sans exercer de discrimination selon la nature de l'acheteur. Le DSGV, mais également des banques privées, ont déjà signalé leur intérêt. Un autre cas va plus loin encore : la WestLB a décidé de vendre ses $27 \%$ de la HSH-Nordbank, une caisse d'épargne appartenant à la fédération des caisses d'épargne du Schleswig-Holstein. Or l'acquisition des $27 \%$ par l'investisseur américain J.C. Flowers est problématique pour le groupe DSGV, étant donné que toute caisse d'épargne est intégrée au fonds d'aides des banques publiques...

Sur fond de tels développements, la question plus générale de la justification du statut public des caisses d'épargnes se posera tôt au tard. Cette justification est fondamentale, car le maintien de ce statut est l'obstacle majeur au rachat de caisses ou de Landesbanken par des opérateurs privés. Le statut est inscrit dans les lois respectives des Länder concernant les caisses d'épargne et les Landesbanken et est basé sur une mission publique. Cependant, cette mission est en train de devenir obsolète ou au moins disproportionnée. Ainsi, un réseau de banques publiques n'est nécessaire ni au soutien à l'esprit d'épargne, ni 
pour fournir des services de banque 'maison' aux collectivités locales. Si néanmoins, on veut retenir l'idée que l'Etat ou les collectivités disposent de leur propre banque pour certaines opérations, l'existence d'établissements spécialisés (à l'instar de la KfW) est préférable. Et elle sera aussi en parfaite adéquation avec les principes de la réglementation européenne qui autorise certaines garanties publiques si elles se limitent à des activités complémentaires au marché (Marktergänzungsfunktion).

Ensuite, l'offre suffisante de crédit au Mittelstand ne dépend pas du caractère public des instituts. II s'agit plutôt de la volonté de s'adapter aux besoins financiers spécifiques de cette clientèle comme le montre la large spécialisation des caisses d'épargne comme des banques coopératives et mutualistes dans ce secteur. Les banques privées ont ici un retard flagrant. Par ailleurs, l'existence d'un réseau de banques publiques n'est pas non plus nécessaire pour assurer, dans l'espace, un large approvisionnement en services bancaires. Cet argument perd de sa pertinence face au développement des TIC. En outre, si le nombre de filiales a fortement chuté (36000 aujourd'hui contre plus de 50000 à la fin 1999), il demeure toujours très élevé.

Enfin, un dernier argument en faveur du soutien public aux caisses d'épargnes est que l'intervention publique contribuerait à maintenir un haut niveau de concurrence. Cependant, cette concurrence n'est pas particulièrement prononcée sur le marché allemand: les deux principales raisons sont l'interdiction de rachat de banques publiques par des investisseurs privés, ainsi que l'application du principe régional (Regionalprinzip), c'est-à-dire la répartition du marché telle qu'elle est pratiquée à l'intérieur des groupes publics et coopératifs. Au lieu d'intervenir dans le jeu de la concurrence par voie statutaire, l'Etat devrait faire confiance au droit de la concurrence et notamment au contrôle des fusions comme il se pratique, avec succès, sur d'autres marchés. D'ailleurs, la Commission des monopoles et le Conseil des Sages (voir REA 78/06) ont signalé à plusieurs reprises leur mécontentement quant à la situation actuelle et prônent un assouplissement du statut des banques publiques.

Compte tenu des réalités politiques et institutionnelles, une mutation du statut des banques publiques paraît néanmoins peu probable, au moins à moyen terme. Elle n'a pas de soutien politique car les législateurs régionaux ne veulent risquer ni de perdre leur pouvoir ni d'entrer en conflit avec les propriétaires locaux (communes et cantons). Mais plus fondamental encore est le problème de la structure fédérale qui rend difficile une réforme cohérente. Certes, un ou plusieurs Länder pourraient jouer une fonction pilote en expérimentant par exemple de nouvelles structures capitalistiques pour les banques publiques. Cependant, le $\S 28$ de la Loi Fondamentale, qui établit le principe de l'autonomie administrative des communes, limite de telles perspectives. L'abandon des garanties publiques en 2005 a néanmoins changé la donne. Ces garanties étaient en effet basées sur le principe que les fondateurs d'un établissement bancaire public doivent toujours veiller à doter l'établissement des moyens financiers requis par ses missions. Ainsi, on peut déduire de la disparition de ces garanties la nécessité d'un changement du statut juridique des caisses (et ainsi de leurs structures capitalistiques).

Une réelle évolution est plus probable si elle est impulsée de l'extérieur. C'est dans ce contexte que s'inscrit la récente requête de la Commission européenne au sujet du $\S 40$ de la Loi bancaire (Kreditwesengesetz) qui stipule que le nom de «Sparkasse » est réservé aux établissements publics. L'occasion est la vente imminente de la Berliner Sparkasse. Selon la Commission, la protection de la «marque » Sparkasse est une violation du droit communautaire, car aucun investisseur ne doit être discriminé par sa raison sociale. Si le gouvernement allemand insiste sur une solution spécifique pour la Berliner Sparkasse, afin d'éviter qu'il ne devienne un précédent risquant la dissolution à terme du système à trois piliers, il suggère néanmoins que tout investisseur
L'approvisionnement en services bancaires est garanti

Le soutien public limite la concurrence

Un changement de statut s'impose

Conflit avec la Commission sur la marque Sparkasse 
puisse à l'avenir préserver le nom de Sparkasse si son engagement intervient dans le cadre d'une procédure de subvention soumise à l'approbation de la Commission. Cependant, cette proposition équivaut à un sophisme, car il n'y a actuellement pas d'autre cas d'aide similaire, et il est très peu probable que la Commission autorisera un tel cas à l'avenir. Compte tenu de cette divergence des points de vue, les deux parties ont décidé d'ajourner les principaux points de discorde, tout en autorisant la vente de la Berliner Sparkasse à un investisseur privé, mais de la considérer comme cas isolé.

MALGRe Les mUtATIONS du CAPITALISme international et national, le système bancaire allemand reste marqué par une forte inertie, corollaire d'une grande stabilité du système financier, parce qu'il se base sur une étroite complémentarité entre structures bancaires et économiques. En contrepartie, il n'est pas parmi les plus rentables, ce qui est en quelque sorte le prix à payer pour un système qui a toujours contribué au bon fonctionnement de l'économie allemande en lui épargnant des crises déstabilisantes, observées dans d'autres systèmes bancaires. Malgré certains atouts, ces derniers ne représentent pas forcément de bons exemples pour les banques allemandes qui restent marquées par une culture de responsabilité collective. Le cas des banques britanniques, certes très rentables, montre que la profitabilité ne rime pas toujours avec une offre qui convienne aux consommateurs, comme l'a constaté récemment l'autorité britannique de la concurrence au sujet des problèmes dans les transactions de paiements ou dans la disponibilité de crédit.

Les banques allemandes ont choisi de moderniser leurs structures afin d'améliorer leur rentabilité : restructurations et spécialisation de l'offre de produits, consolidation interne et externe. Par exemple, la Postbank pourra être un excellent complément aux Landesbanken ou aux banques privées. Ces deux dernières se sensibiliseront davantage aux problèmes des entreprises du Mittelstand afin de réduire leur insuffisance en capitaux propres. Ces évolutions s'inscrivent dans le contexte plus général des mutations de la sphère financière allemande : dans la gouvernance financière et d'entreprise, où le pouvoir de l'actionnaire se renforce, dans un lien entre banque et entreprise qui tend à s'affaiblir, ainsi que dans les règles de comptabilité et du droit financier qui deviennent moins favorables aux emprunteurs. A ceci s'ajoutent les débats sur le statut des banques publiques. Ces mutations peuvent ébranler un système bancaire et financier dont la stabilité actuelle tient justement à la complémentarité entre le système de gouvernance existant, l'orientation des règles comptables et le rôle des banques dans l'économie, à la fois en tant que financiers et 'tuteurs' des entreprises. Loin de basculer vers un système à l'anglo-saxonne, avec prédominance du marché au détriment des banques, et qui risque de provoquer une rupture déstabilisatrice, la modernisation graduelle vise au contraire à préserver le haut degré de complémentarité et de cohésion dont le système économique et financier de l'Allemagne a fait preuve jusqu'ici.

\section{Indications bibliographiques :}

DeUtSCHE BundesbanK, “ Die Ertragslage der deutschen Kreditinstitute », Rapport mensuel, septembre 2006

GABEL M., "Le système bancaire allemand face à la mondialisation - vers la fin d'un modèle ? ", "La finance allemande dans l'impasse immobilière " et " De la banque-industrie aux industries bancaires ", Regards sur l'économie allemande, n66/2004, n64/2003 et $n^{\circ} 45 / 2000$

HACKethal A., Schmidt R. H., TYRell M., " The transformation of the German financial system », Revue d'économie politique, volume 117, juillet-août 2006

MOSCHEL W., "Reformbedarf des deutschen Bankensektors », ifo-Schnelldienst, n²3, 2006

SACHVERSTÄNDIGENRAT, Erfolge im Ausland - Herausforderungen im Inland, Wiesbaden, 2004

«Spezial Banken », Wirtschaftswoche, 26 juin 2006 